\title{
Zur Kenntniss der Eiweissreactionen und von dem Verhalten des Albumins der lichtbrechenden Medien des Auges.
}

Von

\section{Johann Dogiel.}

Hierzu Tafel III.

Fiir den Nachweis des Albumins im Harn und in andern thierischen Flïssigkeiten sind mehrere Reactionen in Vorschlag: gebracht worden, von denen die meisten das Ausfällen des Albumins aus seinen Lösungen zum Ziele haben. Hierher gehören die Reactionen mit starken $\left(\mathrm{NHO}_{3}, \mathrm{H}_{2} \mathrm{SO}_{4}, \mathrm{ClH}\right)$ gleichwie mit schwachen Mineralsäuren (z. B. $\left.\mathrm{CO}_{2}\right)$, mit Metallsalzen $\left(\mathrm{HgCl}_{2}, \mathrm{SCuO}_{4}, \mathrm{SZnO}_{4}\right.$ u. s. w.), Aethylalcohol, Chloroform, Aether und das Erwärmen auf 55 bis $80^{\circ} \mathrm{C}$. Weil aber das Albumin sich nicht immer in gleicher. Weise zu den genannten Reagentien verhält, so nimmt man die Existenz mehrerer Eiweissstoffe an: das Eiweiss des Blatserums, Hühnereiweiss, Kalium- und Natriumalbuminat (Globulin, Kasein), fibrinoplastischer und fibrinogener Stoff, Myosin, Fibrin, Syntonin, Parapepton, Hämoglobin. Die ungleiche Löslichkeit des Eiweisses in Reagentien beruht wahrscheinlich auf der ungleichen Verbindungsweise desselben mit Kalium, Natrium, Calcium und den Säuren. Im Allgemeinen wird das Eiweiss von kaustischen Alkalien gelöst, dagegen von starken Mineralsäuren und der schwachen Kohlensäure zum Coaguliren gebracht. Das Gesagte ist aber nur bis zu einem gewissen Grade richtig, denn wenn einerseits alkalische (Aetzkali) Lösungen von bestimmter Concentration das Eiweiss lösen, so sehen wir andererseits dass eine Kalilösung von $35-40$ p. Ct. nur einige von den Eiweissstoffen löst, dagegen die andern entweder verdichtet oder unverändert lässt. Die rothen Blutscheibchen scheinen durch die Einwirkung einer Kalilange 
von $40 \%$ compakter zu werden und behalten recht lange ihre Form; mit Hilfe einer solchen Lösung gelingt es z. B. leicht die Muskelzellen des Herzens zu isoliren u. s. w. Concentrirte Chlorwassertoffsäure, gleichwie Salpetersäure, coaguliren das Eiweiss, im verdünnten Zustande dagegen lösen sie einige Eiweissstoffe auf. Aber nicht nur der Grad der Concentration der Säuren, sondern auch der des Aethylaleohols ändert das Verhalten des Eiweisses. Aethylalcohol von mindestens 30 p. Ct. Tralles coagulirt das Eiweiss, and ein mehr verdünnter löst dasselbe auf. Eine Temperatur von 60 bis $80^{\circ} \mathrm{C}$. macht das Eiweiss unlöslich, während das Erwärmen auf 50 bis $55^{\circ} \mathrm{C}$. das Auflösen einiger Eiweissstoffe begiinstigt, eine Eigenschaft, von der man in der Histologie beim Isoliren der Muskelelemente Gebrauch macht. Dureh den Zusatz eines Alkalis oder einer Säure zu einer Eiweisslösung, verliert diese die Eigenschaft beim Erwärmen zu coaguliren. Nach den Untersuchungen von A. Schmidt coagulirt das dialysirte Eiweiss nicht durch die Einwirkung des Aethers, was von einem besonderen Salzgehalte dieses Eiweisses abhängen mag. Daher sind aber auch die Methoden, die das Eiweiss durch Coaguliren ausscheiden, für den Nachweis geringer Quantitäten desselben nicht immer zuverlässig.

Eine andere Methode für den Nachweis des Albumins in thierischen Fliissigkeiten besteht in einer Färbung der eiweisshaltigen Lösung durch gewisse Reagentien. Durch Zușatz von Sehwefelsäure und Zucker färbt sich das Eiweiss roth, und durch $\mathrm{KHO}$ and $\mathrm{SCuO}_{4}$ violett. Salpetersaures Quecksilberoxydul (Millon) färbt das Fiweiss beim Erwärmen ziegelroth. Concentrirte Salpetersäure färbt das Eiweiss gelb (Xanthoproteinreaction). Schwefelsäure von 1,8095 specif. Gew. färbt nach den Untersuchungen von Adamkiewicz ${ }^{1}$ ) sehr verschieden: gruin, gelb, orange, roth, violett, je nach der Menge des Eiweisses nnd der Schwefelsäure; gleichzeitig kann man beim auffallenden Lichte deutlich grüne Fluorescenz beobachten.

Die violette Färbung der Eiweisslösung tritt gleichfalls bei Zuṣatz von Essigsäure und concentrirter-Schwefelsäure ein; eine gleiche Reaction ist zu beobachten, wenn statt Eiweiss das Albuminat des Kupfers, Silbers u. s. w. genommen wird. Ueberhaupt

1) Pflügers Archiv f. d. ges. Physiol. Bd. IX. 2. Heft. \$. 156. 
ist aber die Essig-Schwefelsäurereaction sebr" empfindlich, wovon ich mich leicht durch eigene Versuche überzeugen konnte.

Bei der Prüfung der Eiweissreactionen brauchte ich bald Hiihnereiweiss, bald Linsen verschiedener Thiere, bald verschiedene thierische Flüssigkeiten, wie z. B. die seröse Flüssigkeit aus dem Pericardium, humor aqueus, Lymphe u. s. w. Von den Säuren gebrauchte ich: acidum aceticum glaciale, Chlorwasserstoffsäure von 1,2 specif. Gew., Schwefelsäure von 1,809 specif. Gew. und rauchende Salpetersäure; die Säuren waren chemisch rein.

Beim Behandeln der Linse mit Essigsäure und nachträglich mit Schwefelsäure ist eine intensive violette Färbung, die auch durch Chlorwasserstoffsäure im Gemische mit Schwefelsäure, oder, beim Erwärmen, durch rauchende Chlorwasserstoffsäure allein erzeugt wird, zu beobachten. Ausserdem gelang es mir die Beobachtung zu machen, dass die Linse (von Hund, Katze, Kaninchen, Frosch), die vorläufig in Essigsäure gelegen hat, durch Zusatz von Chlorwasserstoffsäure und durch eine mehrtägige Einwirkung der freien Luft sich blau und bald darauf violett färbte. Das Gleiche beobachtet man bei der Einwirkung der Luft und rauchender Chlorwasserstoffsäure.

Um im gegebenen Falle den Grad des Einflusses des Sauerstoffs der Luft zu bestimmen, leitete ich Sauerstoff, gleichwie Ozon, durch eine Lösung der Linse in Essig-Chlorwasserstoffsäure. Das Ozon stellte ich zu diesem Zwecke aus reinem und vollkommen trockenem Sauerstoffe dar, indem ich den letztern in den Siemens'schen Apparat leitete und der Einwirkung einer funkenlosen Entladung eines starken Ruhmkorff'schen Apparats, der mit zwölf Bunsen'schen Elementen in Verbindung stand, aussetzte. In Folge der Einwirkung des Ozons auf die essig-chlorwasserstoffsaure Eiweisslösung färbte sich diese grün, blau, violett, roth und zuletzt gelb. Diese Reaction findet aber nicht inmer in gleicher Weise statt, indem sich die Lösung im Anfange bald grün, bald blau, bald selbst gelb färbt. Fügt man zur Lösung der Linse verdünnte Essig-Chlorwasserstoffsäure hinzu, und leitet erst dann durch die Lösung Ozon, so stellt sich sehr oft blaue Färbung ein, was auch in dem Falle zu beobachten ist, wenn statt Ozon, Sauerstoff in eine Lösung des Eiweisses in Eisessig und rauchender Chlorwasserstoffsäure geleitet wird. Dagegen tritt gewöhnlich citronengelbe Färbung ein, wenn die Lösung des Eiweisses in rauchender 
Chlorwasserstoffsäure mit Ozon behandelt wird. Einer längern Einwirkung des letztern ausgesetzt, nimmt die Lösung eine Farbe an, die der des Pikrokarmins nicht unähnlich ist. Um die ganze Farbenreihe von grïn bis gelbzu erhalten, braucht man nur die mechanisch zerkleinerte Linse so lange mit 0 zon (etwa eine halbe bis eineStunde) in Eisessig z u behandeln, bis sich eine citronengelbe Färbung einstellt; wenn man dann rauchende Chlorwasserstoff äure hinzufügt und die Lösung einige Zeit stehen lässt, so stellen sich allmählig der Reihe nach die früher genannten Farben ein (Fig. 9). Bemerkenswerth ist der Umstand, dass nachdem man die Linse in Wasser zerkleinert und Ozon durch die Flüssigkeit geleitet hat, sich dieselbe etwa nach einer Stunde trubt, indem sich Eiweiss in Form einer zähen Masse, die an Fibrin erinnert, ausscheidet. Wenn man zu diesem Eiweiss Essig-Chlorwasserstoffäure hinzusetzt und 0 zon hindurchleitet, so erhält man nicht die früher genannten Farben.

Aus dem Gesagten folgt, dass bei der Einwirkung des gewöhnlichen Sauerstoffs, gleichwie des Ozons auf das Eiweiss der Linse in Gegenwart der Essigsäure oder Essig-Chlorwasserstoffsäure, sich das Eiweiss sehr verschieden färbt, und dass diese Färbung von der Oxydation des Eiweisses abhängt. Dagegen findet eine derartige Färbung nicht statt, wenn vor der Behandlung mit den genannten Säuren das Eiweiss der Wirkung des Ozons ansgesetzt wird. Der höchste Grad der Oxydation des Eiweisses in Gegenwart der Essig-Chlorwasserstoffsäure wird durch gelbe Färbung angezeigt, eine Reaction, die sehr viel Aehnlićbkeit mit der Xanthoproteinreaction hat, oder auch mit der durch concentrirte Säure hervorgerufenen. Mit einem Worte, die Veränderung der Farbe des Eiweisses durch Salpetersäure, Schwefelsäure, oder auch durch Essig-Chlorwasserstoffsäure, wird. wesentlich durch verschiedene Grade der Oxydation des Eiweisses bedingt.

Auf den beigefügten Zeichnungen sieht man nur einen Theil der Farben, die durch die Einwirkung des Ozons auf eine Lösung der Linse in Eisessig und Chlorwasserstoffsäure der Reihe nach. hervorgerufen werden. Bemerkt muss hier werden, dass während der Farbenänderung es leicht gelingt beim auffallenden Lichte das Fluoresciren der Lösung, ganz wie bei der Einwirkung con- 
centrirter Schwefelsäure auf das Eiweiss (Adamkiewicz), zu beobachten.

Indem ich die Reaction des Eiweisses mit Essigsäure, Schwefelsäure und andern Reagentien benutzte, stellte ich eine Reihe von Versuchen an, die das Verhalten des Eiweisses der lichtbrechenden Medien des Auges zur Aufgabe hatten. Zu diesen Medien gehören: die Hornhaut, humor aqueus, die Linse und der Glaskörper. Bei der Einwirkung der Essig-Schwefelsäure auf den ganzen centralen Theil der Hornhaut sowie auf das äussere Epithelium derselben, auf die substantia propria cornea und das Endothel der Descemetischen Haut erhält man eine charakteristische violette Färbung, die die Anwesenheit des Eiweisses anzeigt. Nach Laptschinsky ${ }^{1}$ ) enthält die Augenlinse ungefähr 35\% Eiweissstoffe, von denen im gegebenen Falle als verschieden Serumalbumin und Globulin genannt werden. Von der Wirkung verschiedener Säuren auf die Linse ist schon früher die Rede gewesen. In Bezug auf humor aqueus ist zu bemerken, dass ausser Wasser, Salze und Zucker, auch Eiweiss als einer seiner Bestandtheile genannt wird. Durch eigene Versuche, die ich an lebenden und todten Thieren anstellte, konnte ich mich in der. That von dem Vorhandensein dieses Bestandtheils tiberzengen.

Um beim Sammeln des humor aqueus die Linse nicht zu verletzen, durchstach ich vorsichtig die Hornhaut mit einer Staarnadel. Die Quantität des Eiweisses im humor aqueus versehiedener Thiere (Kalb, Hund, Kaninchen, Hecht, Frosch) ist so gering, dass ich Anfangs Gefahr lief dasselbe gänzlich zu übersehen. Sammelt man aber den humor aqueus eines grossen Hundes oder eines Kalbes im Probecylinder und fügt einige $e_{c}$ Tropfen Eisessig and mit gehöriger Vorsicht Schwefelsäure von 1,8 specif. Gewicht hinzu, so bildet sich nach einiger Zeit auf der Grenze zwischen der Schwefelsäure und dem übrigen Theil der Flüssigkeit ein violetter Ring (Fig. 21).

Was endlich den Glaskörper betrifft, so konnte in demselben mit Hilfe verschiedener Reagentien nur eine Spur von Eiweiss nachgewiesen werden. Da aber diese geringe Quantität stets vorhanden ist, so istkaum anzunehmen, dass sie von einer Beimischung des Eiweisses

1) Ein Beitrag zur Chemie des Linsengewebes. Von Dr. Laptschinsky. Pflüger's Archiv. Bd. XIII. Heft 12. 1876. 
der Retina oder der Linse herriihrt. Zum humor aqueus zuriekkehrend, will ich hier bemerken, dass diese Flüssigkeit ihrer Zusammensetzung und Entstehungsweise nach von Vielen mit der Lymphe identificirt wird. Diejenigen Forscher, die der Ansicht sind, dass im Allgemeinen die Quantität der Lymphe im direkten Verhältniss zum Blutdrucke steht, suchen auch die Verschiedenheit in der Quantität wie in der Qualität des humor aqueus durch das Schwanken des Blutdrucks zu erklären. Um sich von der Richtigkeit dieser Annahme zu überzeug'en suchte ich die Veränderung im Blutdrucke künstlich hervorzurufen und den bei verschiedenem Drucke erhaltenen humor aqueus annähernd auf seinen Gehalt an Eiweiss zu. prüfen, wobei die Intensität der Färbung, die durch Essig-Schwefelsäure erzeugt wird, zum Anhaltspunkte diente.

Sollte humor aqueus Lymphe sein, die in den Gefässen der Ciliarfortsätze und der Iris entsteht, so wäre zu erwarten, dass mit der Aend erung der Blutcireulation in den genannten Theilen des Auges sich auch die Zusammensetzung und Quantität des humor aqueus ändern würde. $\mathrm{Zu}$ Gunsten einer Aenderung der Quantität des Eiweisses in der Flüssigkeit der Vorderkammer des Auges spricht die Beobachtung (Adamük), dass beim Ausfliessen aus einer Oeffnung in der Hornhaut diese Flüssigkeit unter gewissen Umständen coagulirt. Ausserdem soll auf Grund der im Laboratorium des Professors Gruenhagen in Königsberg gemachten Beobachtungen ${ }^{1}$ ) die Quantität dieser Flüssigkeit vom Blutdrucke abhängen.

Bei meinen Versuchen wurde der humor aquens aus dem einen Auge des lebenden Thieres (Hund) bei normalem, und aus dem andern Auge bei künstlich verändertem Blutdrucke gesammelt, und die Quantität des Eiweisses nach der oben erwähnten Methode bestimmt.

Die Veränderung im Blutdrucke wurde durch folgende Mittel hervorgerufen: 1) durch Unterdrückung and Wiederherstellung der Athmung, 2) durch das Unterbinden der Aorta descendens oberhalb des Zwerchfells, 3) durch Injection von starkem Aethylalkohol in das peripherische Ende der art. carotis (wobei der Blutdruck von 116 bis $200 \mathrm{~mm} \mathrm{Hg}$. stieg), 4) durch Injection von $\mathrm{Cl}_{2} \mathrm{Ba}$ in die Venen der hintern Extremitäten, 5) durch Vergiftung mit

1) Ueber die Secretion des humor aqueus, etc. 
Kohlenoxyd, Atropin, Chlorhydrat. Aber alle in dieser Richtung gemachten Versuche gaben ein negatives Resultat, da die Quantitat des Eiweisses im humor aqueus sich unabhängig rom Blutdrucke zeigte.

Wenn auch die Quantität der Flüssigkeit der Vorderkammer des Auges mit dem Blutdrucke steigt oder fällt, wie dieses Chabas behauptet, so kann möglicher Weise diese Erseheinung, unabhängig vom Blutdrucke, dureh andere Ursachen hervorgerufen werden, da ja auch die Quantität der Lymphe, nach den Beobachtungen vieler Forscher, bald mit dem Blutdrucke steigt, bald aber auch nicht.

Besonders sind es aber die Untersuchungen von Paschutin und Eminghausen, die uns belehren, dass zwischen der Quantität der ausfliessenden Lymphe und der Stärke des Blutdrucks kein direkter Zusammenhang stattfindet. Zu ähnlichen Resultaten gelangte auch Holtzmann durch die von ihm in meinem Laboratorium angestellten Versuche, die bald veröffentlicht werden sollen.

Wenn man also die vordere Augenkammer als einen Lymphraum, dessen Inhalt Lymphe ist, betrachtet, so muss man nach dem oben Mitgetheilten zu dem Schlusse kommen, dass auch auf den besagten Inhalt der Blutdruck keinen direkten Einfluss ausübt; übrigens ist man kaum berechtigt, wie dieses schon früher erwähnt wurde, humor aqueus mit der Lymphe zu identificiren, da in der Zusammensetzung dieser Flüssigkeiten ein bedeutender Unterschied stattfindet.

\section{Erklärung der Abbildungen anf Taf. III.}

Fig. 1 bis 9 zeigen die Farben, die durch die Einwirkung verschiedener Reagentien auf Eiweisslösungen erzeugt werden, und zwar:

Fig. 1. durch Zucker und Schwefelsäure.

Fig. 2. durch die Millon'sche Probe.

Fig. 3. Uurch kaustisches Kali und Kupfervitriol (Piotrowsky).

Fig. 4. durch Salpetersäure.

Fig. 5. durch concentrirte Schwefelsäure (Adamkiewicz). 
Fig. 6. durch Chlorwasserstoffsäure und Erwärmen.

Fig. 7. Die Farbe derselben Lösung nach dem Erkalten.

Fig. 8. Die Farbe der anfangs erwärmten und später erkalteten chlorwasserstoff-schwefelsauren Lösung.

Fig. 9 durch Essigsäure und Ozon, und späteres Behandeln mit Chlorwasserstoffsäure.

Fig. 10, 11, 12. Die Farbenveränderung des Eiweisses der Linse durch Chlorwasserstoffsäure und Qzon.

Fig. 13, 14, 15, 16, 17 und 18. Die allmählige Farbenveränderung des Eiweisses der Linse durch Essig-Chlorwasserstoffsäure und Ozon.

Fig. 19. Die Farbe, die durch die Einwirkung des Ozons und der Chlorwasserstoffsäure auf die essigsaure Lösung der Linse des Hechtes erzeugt wird.

Fig. 20. Farbe derselben Lösung nach längerer Einwirkung des Ozons.

Fig. 21. Farbe des humor aqueus mit Essig-Schwefelsäure.

\title{
Ueber den Nachweis des Lecithins.
}

\author{
Von
}

\section{Oscar Loew,}

Adjunct am pflanzenphysiologischen Institut zu München.

In einer von Prof. C.v.Nägeli und mir publicirten Arbeit ${ }^{1}$ ) über die Bestandtheile der Hefe habe ich erwähnt, dass die Hefe, entgegen den Angaben Hoppe-Seylers, kein Lecithin enthalte. Ich hatte kein Mittel unversucht gelassen, die Gegenwart desselben darzuthun und da meine Erwartung getäuscht wurde, meine Aufmerksamkeit anf den Nachweis der characteristischen Zersetzungsproducte - Cholin und Glycerinphosphorsäure - gerichtet, doch war das Resultat kein giinstigeres.

Die Anwesenheit des Lecithins auch in den niederen Pilzen wäre mir von besonderem Interesse gewesen, da diese Substanz

1) Sitzungsber. d. bair. Acad. d. Wissensch. Mai 1878. Liebigs Ann. Bd. 193. Journ. f. pr. Chem. 1878. Durch ein Versehen wurde mein Name in letzterem Journal nicht in der Ueberschrift erwähnt. 


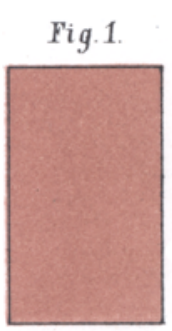

Fig. 1 .

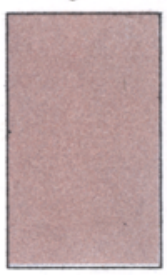

Fig.13.

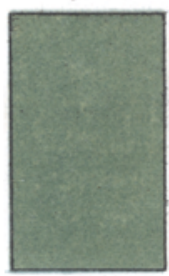

Fig. 2

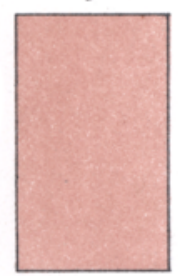

Fig. 8.

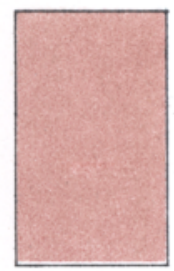

Fig. 14.

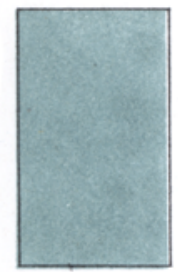

Fig. 19.

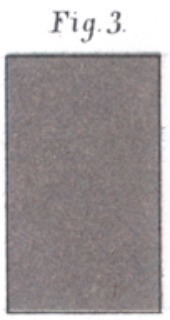

Fig 9

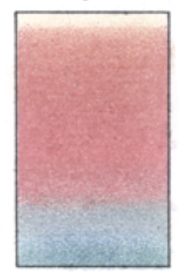

Fig. 15.

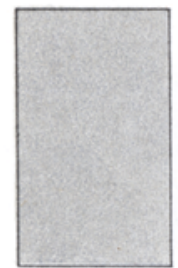

Fig. 20.
Fig.4.

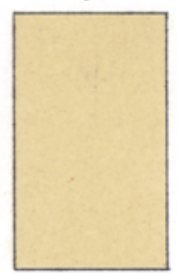

Fig. 10.

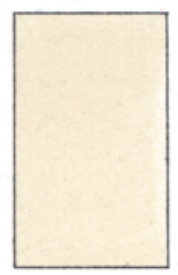

Fig.16.

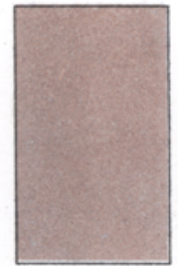

Fig. 21 .
Fig. 5.

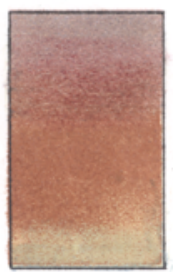

Fig. I1.

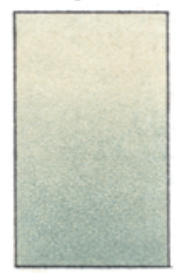

Fig. 17

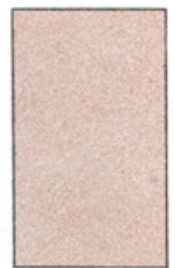

Fig.6.

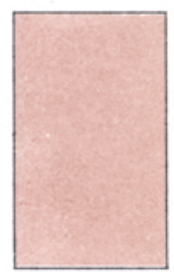

Fig. 12

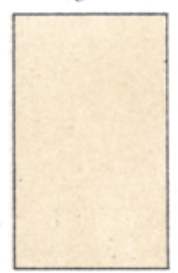

Fig. 18
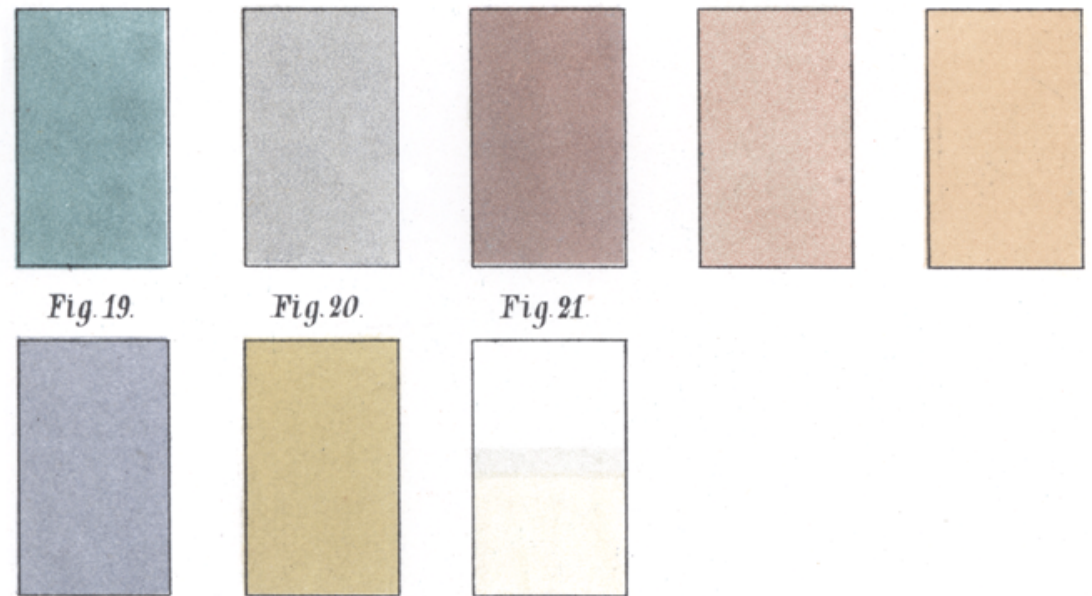